\title{
Monoamine reuptake inhibitors enhance the discriminative state induced by cocaine in the rat
}

\author{
Kathryn A. Cunningham and Patrick M. Callahan \\ Department of Pharmacology and Toxicology, University of Texas Medical Branch, Galveston, TX 77550, USA
}

The legend to Fig. 2 on p. 179 should be corrected as follows:

Fig. 2. Results of substitution tests with monoamine reuptake inhibitors. Desipramine (diamonds), fluoxetine (squares), or GBR 12909 (triangles) was administered during substitution tests at the doses indicated. For all doses of desipramine and fluoxetine, the percent drug-lever responding was significantly different than the previous cocaine session; asterisks were omitted for clarity. Symbols as in Fig. 1. Data points are the mean of $8 / 8$ rats 